\title{
The Tarski Theorems and Elementary Equivalence of Group Rings
}

\author{
Benjamin Fine1, Anthony Gaglione², Gerhard Rosenberger ${ }^{3}$, Dennis Spellman ${ }^{4}$ \\ ${ }^{1}$ Department of Mathematics, Fairfield University, Fairfield, CT, USA \\ ${ }^{2}$ Department of Mathematics, United States Naval Academy, Annapolis, MD, USA \\ ${ }^{3}$ Fachbereich Mathematik, University of Hamburg, Hamburg, Germany \\ ${ }^{4}$ Department of Statistics, Temple University, Philadelphia, PA, USA \\ Email: fine@fairfield.edu
}

How to cite this paper: Fine, B., Gaglione, A., Rosenberger, G. and Spellman, D. (2017) The Tarski Theorems and Elementary Equivalence of Group Rings. Advances in Pure Mathematics, 7, 199-212.

https://doi.org/10.4236/apm.2017.72011

Received: November 23, 2016

Accepted: February 11, 2017

Published: February 14, 2017

Copyright (C) 2017 by authors and Scientific Research Publishing Inc. This work is licensed under the Creative Commons Attribution International License (CC BY 4.0).

http://creativecommons.org/licenses/by/4.0/ (c) (i) Open Access

\begin{abstract}
The Tarski theorems, proved by Myasnikov and Kharlampovich and independently by Sela say that all nonabelian free groups satisfy the same firstorder or elementary theory. Kharlampovich and Myasnikov also prove that the elementary theory of free groups is decidable. For a group ring they have proved that the first-order theory (in the language of ring theory) is not decidable and have studied equations over group rings, especially for torsionfree hyperbolic groups. In this note we examine and survey extensions of Tarksi-like results to the collection of group rings and examine relationships between the universal and elementary theories of the corresponding groups and rings and the corresponding universal theory of the formed group ring. To accomplish this we introduce different first-order languages with equality whose model classes are respectively groups, rings and group rings. We prove that if $R[G]$ is elementarily equivalent to $S[H]$ then simultaneously the group $G$ is elementarily equivalent to the group $H$ and the ring $R$ is elementarily equivalent to the ring $S$ with respect to the appropriate languages. Further if $G$ is universally equivalent to a nonabelian free group $F$ and $R$ is universally equivalent to the integers $\mathbb{Z}$ then $R[G]$ is universally equivalent to $\mathbb{Z}[F]$ again with respect to an appropriate language.
\end{abstract}

\section{Keywords}

Group Ring, Elementary Equivalent, Universally Equivalent, Direct Union, Discriminates

\section{Introduction}

For a general algebraic object $A$, for example a group, a ring, a field or an 
algebra, its elementary theory is the set of all first-order sentences in a logical language appropriate for that object, true in $A$ (see Section 2). Hence if $F$ is a given free group, its elementary theory consists of all first-order sentences in a language appropriate for group theory that are true in $F$. Two algebraic objects are elementary equivalent or elementarily equivalent if they have the same elementary theory. The Tarski theorems proved by Kharlampovich and Myasnikov and independently by Sela (see [1] and [2] and the references therein) say that all nonabelian free groups satisfy the same elementary theory. Kharlampovich and Myasnikov also showed that the elementary theory of free groups is decidable, that is, there is an algorithm to decide if any elementary sentence is true in all free groups or not. For a group ring they have proved that the firstorder theory (in the language of ring theory) is not decidable and have studied equations over group rings especially for torsion-free hyperbolic groups (see (2)).

The set of universal sentences (see Section 2) in an algebraic object $A$ that are true in $A$ is its universal theory while two objects are universally equivalent if they have the same universal theory. It is straightforward to show that all nonabelian free groups have the same universal theory. As part of the general solution to the Tarski theorems it was shown that a finitely generated nonabelian group is universally free (that is has the same universal theory as a nonabelian free group) if and only if it is a limit group.

In this note, following the studies by Kharlampovich and Myasnikov on equations over group rings [3], we examine and survey extensions of Tarksi-like results to the collection of group rings and examine relationships between the universal and elementary theories of the corresponding groups and rings and the corresponding universal theory of the formed group ring. To accomplish this we introduce different first-order languages with equality whose model classes are respectively groups, rings and group rings. We prove that if $R[G]$ is elementarily equivalent to $S[H]$ then simultaneously the group $G$ is elementarily equivalent to the group $H$ and the ring $R$ is elementarily equivalent to the ring $S$ with respect to the appropriate languages. Further if $G$ is universally equivalent to a nonabelian free group $F$ and $R$ is universally equivalent to the integers $\mathbb{Z}$ then $R[G]$ is universally equivalent to $\mathbb{Z}[F]$ again with respect to an appropriate language.

The outline of this paper is as follows. In the next section we review the basic material on elementary and universal theory. In Section 2 we introduce three first-order languages upon which the results are based. In Section 3 we describe the Tarski theorems and some important related results. In Section 4 we consider the universal theories of group rings while in Section 5 the elementary theories of group rings.

\section{Elementary and First-Order Theories}

Alfred Tarski in 1940 made three well-known conjectures concerning nonabelian free groups. We call these the Tarski Problems or Tarski Conjectures 
and they asked, among other things, whether all nonabelian free groups satisfy the same first-order or elementary theory. This was proved in the affirmative by Kharlampovich and Myasnikov ([4] [5] [6] [7] [8]) and independently by Sela ([9] [10] [11] [12] [13])). We now explain the terminology.

In order to discuss universal and elementary theory for an algebraic object $A$ we must first define appropriate logical languages to deal with the object. In the context of this paper we need three languages; one for groups, one for rings and one for group rings. To this end we introduce three first-order languages with equality that we will call $L_{0}, L_{1}, L_{2}$; the first appropriate for group theory, the second for ring theory and the third for the theory of group rings. We then list sets of axioms $T_{0}, T_{1}, T_{2}$ expressed in $L_{0}, L_{1}, L_{2}$ respectively and and view a group as a model of $T_{0}$ a ring as a model of $T_{1}$. Moreover, we view the class of group rings as a subclass of the model class of $T_{2}$.

We first review the elementary and universal theory of groups. A first-order sentence in group theory has logical symbols $\forall, \exists, \vee, \wedge, \sim$ but no quantification over sets. A first-order theorem in a group $G$ is a theorem that says a firstorder sentence is true in $G$. For a class of groups $\mathcal{G}$ a first-order theorem is a first-order sentence true in all groups in $\mathcal{G}$. We make this a bit more precise:

We start with a first-order language $L_{0}$ appropriate for group theory. $L_{0}$ is the first-order language with equality containing a binary operation symbol $\cdot$ a unary operation symbol ${ }^{-1}$ and a constant symbol 1 . A universal sentence of $L_{0}$ is one of the form $\forall \bar{x}\{\phi(\bar{x})\}$ where $\bar{x}$ is a tuple of distinct variables, $\phi(\bar{x})$ is a formula of $L_{0}$ containing no quantifiers and containing at most the variables of $\bar{x}$. Similarly an existential sentence is one of the form $\exists \bar{x}\{\phi(\bar{x})\}$ where $\bar{x}$ and $\phi(\bar{x})$ are as above. A universal-existential sentence is one of the form $\forall \bar{x} \exists \bar{y}\{\phi(\bar{x}, \bar{y})\}$. Similarly defined is an existential-universal sentence. It is known that every sentence of $L_{0}$ is logically equivalent to one of the form $Q_{1} x_{1} \cdots Q_{n} x_{n} \phi(\bar{x})$ where $\bar{x}=\left(x_{1}, \cdots, x_{n}\right)$ is a tuple of distinct variables, each $Q_{i}$ for $i=1, \cdots, n$ is a quantifier, either $\forall$ or $\exists$, and $\phi(\bar{x})$ is a formula of $L_{0}$ containing no quantifiers and containing free at most the variables $x_{1}, \cdots, x_{n}$. Further vacuous quantifications are permitted. Finally a positive sentence is one logically equivalent to a sentence constructed using (at most) the connectives $\vee, \wedge, \forall, \exists$.

If $G$ is a group then the universal theory of $G$ consists of the set of all universal sentences of $L_{0}$ true in $G$. We denote the universal theory of a group $G$ by $T h_{\forall}(G)$. Since any universal sentence is equivalent to the negation of an existential sentence it follows that two groups have the same universal theory if and only if they have the same existential theory. Two groups $G$ and $H$ that have the same universal equivalently existential theory are said to be universally equivalent.

The set of all sentences of $L_{0}$ true in $G$ is called the first-order theory or the elementary theory of $G$. We denote this by $T h(G)$. We note that being firstorder or elementary means that in the intended interpretation of any formula or sentence all of the variables (free or bound) are assumed to take on as values 
only individual group elements-never, for example, subsets of, nor functions on, the group in which they are interpreted.

We say that two groups $G$ and $H$ are elementarily equivalent (symbolically $G \equiv H)$ if they have the same first-order theory, that is $\operatorname{Th}(G)=\operatorname{Th}(H)$.

Two very important concepts in the elementary theory of groups, are completeness and decidability. Given a nonempty class of groups $\mathcal{X}$ closed under isomorphism we say that its first-order theory is complete if given a sentence $\phi$ of $L_{0}$ either $\phi$ is true in every group in $\mathcal{X}$ or $\phi$ is false in every group in $\mathcal{X}$. The first-order theory of $\mathcal{X}$ is decidable if there exists a recursive algorithm which, given a sentence $\phi$ of $L_{0}$, decides whether or not $\phi$ is true in every group in $\mathcal{X}$. Kharlampovich and Myasnikov (see [1] [2]) proved that the elementary theory of free groups is decidable.

Let $L_{0}$ be the first-order language as above and let $T_{0}$ be the axioms

$$
\begin{aligned}
& \forall x, y, z((x \cdot y) \cdot z=x \cdot(y \cdot z)) \\
& \forall x(x \cdot 1=x) \\
& \forall x\left(x \cdot x^{-1}=1\right) .
\end{aligned}
$$

Then we view groups as models of $T_{0}$, that is the model class of $T_{0}$ is the class of groups.

In order to extend some of these results to group rings we introduce two additional first-order languages $L_{1}$ and $L_{2}$. We then give axioms in these languages so that the models of the axiomatic systems are rings and group rings respectively.

Let $L_{1}$ be the first-order language with equality appropriate for ring theory. The language $L_{1}$ contains two binary operation symbols + and $\cdot$, two unary operation symbols - and ${ }^{-1}$ and two constant symbols 0 and 1 . A ring with unity will then be a model of the following set $T_{1}$ of axioms.

$$
\begin{aligned}
& \forall x, y(x+y=y+x) \\
& \forall x, y, z((x+y)+z=x+(y+z)) \\
& \forall x(x+0=x) \\
& \forall x(x+(-x)=0) \\
& \forall x, y, z((x \cdot y) \cdot z=x \cdot(y \cdot z)) \\
& \forall x(x \cdot 1=x) \\
& \forall x(1 \cdot x=x) \\
& \forall x, y\left(((x \cdot y=1) \wedge(y \cdot x=1)) \rightarrow\left(x^{-1}=y\right)\right) \\
& \forall x\left(\forall y((x \cdot y \neq 1) \vee(y \cdot x \neq 1)) \rightarrow\left(x^{-1}=0\right)\right) \\
& \forall x, y, z(x \cdot(y+z)=x \cdot y+x \cdot z) \\
& \forall x, y, z((x+y) \cdot z=x \cdot z+y \cdot z)
\end{aligned}
$$




$$
1 \neq 0
$$

Thus, we view rings with multiplicative identity $1 \neq 0$ as models of $T_{1}$.

We now let $L_{2}$ be the first-order language with equality containing two binary operation symbols + and $\cdot$, two unary operation symbols - and $^{-1}$, two constant symbols 0 and 1 and two unary relation symbols $\Gamma$ and $P$.

To clarify the relation symbols we wish to model a group ring $R[G]$. The relation symbol $\Gamma$ will identify elements in $G$ and the relation symbol $P$ identifies elements in $R$. Hence $\Gamma(x)$ is true if $x \in G$ and $P(x)$ is true if $x \in R$.

Now we let $T_{2}$ be the union of $T_{1}$ and the following additional axioms.

$$
\begin{aligned}
& \Gamma(1) \\
& \forall x, y((\Gamma(x) \wedge \Gamma(y)) \rightarrow \Gamma(x \cdot y)) \\
& \forall x(\Gamma(x) \rightarrow \exists y(\Gamma(y) \wedge(x \cdot y=1) \wedge(y \cdot x=1))) \\
& P(0) \\
& P(1) \\
& \forall x, y((P(x) \wedge P(y)) \rightarrow(P(x+y))) \\
& \forall x, y((P(x) \wedge P(y)) \rightarrow P(x \cdot y)) \\
& \forall x(P(x) \rightarrow P(-x)) \\
& \forall x\left(P(x) \rightarrow P\left(x^{-1}\right)\right) \\
& \forall x, y(P(x) \rightarrow x \cdot y=y \cdot x)
\end{aligned}
$$

If $A$ is a model of $T_{2}$, we let $\Gamma(A)=\{a \in A: \Gamma(a)\}$ and $P(A)=\{a \in A: P(a)\}$.

Thus, $\Gamma(A)$ is a group and $P(A)$ is a commutative ring with identity $1 \neq 0$ which is central in $A$. We call a model $A$ of $T_{2}$ standard provided every element $a \in A$ is uniquely expressible in the form $\sum_{g \in \Gamma(A)} r(g) \cdot g$ where $r(g) \in P(A)$ for all $g \in \Gamma(A)$ and at most finitely many $r(g)$ are nonzero. Thus, we view group rings as standard models of $T_{2}$. Henceforth we omit the binary operation symbol · in favor of juxtaposition.

With these languages and model classes in place we can speak of universal equivalence and elementary equivalence of rings and group rings. Hence two rings $R, S$ are universally equivalent if they satisfy the same universal sentences relative to $L_{1}$ and elementary equivalent if they satisfy the same first-order sentences relative to $L_{1}$. Analogously two group rings $R[G], S[H]$ are universally equivalent if they satisfy the same universal sentences relative to $L_{2}$ and elementary equivalent if they satisfy the same first-order sentences relative to $L_{2}$.

\section{The Tarski Theorems in Groups}

Alfred Tarski made three well-known conjectures concerning the first-order 
theory of nonabelian free groups. The purpose of this paper is to extend if possible parts of these to group rings. Here we recall these basic results. Complete discussions can be found in the book [1] and in the paper [2].

Tarski first asked the general question whether all nonabelian free groups share the same elementary theory. Vaught, a student of Tarksi's, proved almost immediately that all free groups of infinite rank do have the same elementary theory, and thus reduced the question to the class of nonabelian free groups of finite rank. After this, Tarski's question was formalized into the following conjectures.

Tarski Conjecture 1. Any two nonabelian free groups are elementarily equivalent. That is any two nonabelian free groups satisfy exactly the same firstorder theory.

Tarski Conjecture 2. If the nonabelian free group $H$ is a free factor in the free group $G$ then the inclusion map $H \rightarrow G$ is an elementary embedding.

ASn elementary embedding is a group monomorphisms which preserves the truth of first-order formulas. Specifically, if $H$ and $G$ are groups and

$$
f: H \rightarrow G
$$

is a monomorphism then $f$ is an elementary embedding provided whenever $\phi\left(x_{0}, \cdots, x_{n}\right)$ is a formula of $L_{0}$ containing free at most the distinct variables $x_{0}, \cdots, x_{n}$ and $\left(h_{0}, \cdots, h_{n}\right) \in H^{n+1}$ then $\phi\left(h_{0}, \cdots, h_{n}\right)$ is true in $H$ if and only if

$$
\phi\left(f\left(h_{0}\right), \cdots, f\left(h_{n}\right)\right)
$$

is true in $G$. If $H$ is a subgroup of $G$ and the inclusion map $i: H \rightarrow G$ is an elementary embedding then we say that $G$ is an elementary extension of $H$. It follows that the second conjecture is stronger than the first and in fact implies the first. If true, then the theory of the nonabelian free groups would be complete, that is given a sentence $\phi$ of $L_{0}$ then either $\phi$ is true in every nonabelian free group or $\phi$ is false in every nonabelian free group.

Two very important concepts in the elementary theory of groups, are completeness and decidability. Given a non-empty class of groups $\mathcal{X}$ closed under isomorphism then we say its first-order theory is complete if given a sentence $\phi$ of $L_{0}$ then either $\phi$ is true in every group in $\mathcal{X}$ or $\phi$ is false in every group in $\mathcal{X}$. The first-order theory of $\mathcal{X}$ is decidable if there exists a recursive algorithm which, given a sentence $\phi$ of $L_{0}$ decides whether or not $\phi$ is true in every group in $\mathcal{X}$. The third Tarski conjecture was about decidability.

Tarski Conjecture 3. The elementary theory of the nonabelian free groups is decidable.

After a long series of partial results (see [1] [2]) the positive solution to the Tarksi conjectures (1) and (2) was given by $O$. Kharlampovich and $A$. Myasnikov ([3] [4] [5] [6] [7]) and independently by Z. Sela ([8] [9] [10] [11] [12]). The proofs by both sets of authors involved the development of whole new areas of mathematics, in particular an algebraic geometry (Sela calls this diophantine 
geometry) over free groups. In addition Kharlampovich and Myasnikov proved Tarski Conjecture (3).

Theorem 3.1. (1) Any two nonabelian free groups are elementarily equivalent. That is any two nonabelian free groups satisfy exactly the same first-order theory.

(2) If the nonabelian free group $H$ is a free factor in the free group $G$ then the inclusion map $H \rightarrow G$ is an elementary embedding.

(3) The elementary theory of the nonabelian free groups is decidable.

Although Tarksi was never explicit on the origin of the basic question, it is motivated by several basic results, and concepts, in the theory of free groups (see [1] and [14] for complete discussions of free groups). First is the observation that most free group properties, involving elements, are rank independent, that is, true for all free groups independent of rank. For example all nonabelian free groups are torsion-free and all abelian subgroups of nonabelian free groups are cyclic.

A second possible motivation, which also shows that all nonabelian free groups have the same universal theory, is the following. Let $F_{2}$ be a free group of rank 2. It is a straightforward consequence of the Reidemeister-Schreier process (see $[\mathrm{MKS}]$ ) that the commutator subgroup of $F_{2}$ is free of infinite rank. This implies that if we let $F_{\omega}$ denote a free group of countably infinite rank, then $F_{\omega} \leq F_{2}$. That is, $F_{\omega}$ can be embedded isomorphically as a subgroup of $F_{2}$. It follows that for any $m, n \geq 2$ with $m<n$ we have

$$
\cdots \leq F_{\omega} \leq F_{2} \leq F_{m} \leq F_{n} \leq \cdots \leq F_{\omega} \leq \cdots
$$

This shows that $F_{n}$ can be embedded isomorphically in $F_{m}$ and $F_{m}$ can be embedded isomorphically in $F_{n}$. Its like a snake eating its tail.

If $G \subset H$ then any universal sentence in $H$ must also be true in $G$, that is $T h_{\forall}(H) \subset T h_{\forall}(G)$. This observation combined with the observations above prove that all nonabelian free groups have the same universal theory and hence are universally equivalent.

Theorem 3.2. All nonabelian free groups are universally equivalent.

Gaglione and Spellman [15] and independently Remeslennikov [16] gave a characterization of the universally free groups. This characterization became one of the cornerstiones of the proof of the Tarksi theorems. Recall group $G$ is residually free provided, for every $g \in G \backslash\{1\}$, there is a homomorphism $\varphi: G \rightarrow F$, where $F$ is a free group, such that $\varphi(g) \neq 1$. $G$ fully residually free provided, for every finite set of nontrivial elements $g_{1}, \cdots, g_{n} \in G \backslash\{1\}$, there is a homomorphism $\varphi: G \rightarrow F$, where $F$ is a free group, such that $\varphi\left(g_{i}\right) \neq 1$. We also say that $G$ is discriminated by a free group. Finitely generated fully residually free groups are also known as limit groups since they arise as limits of homomorphisms into free groups (see [1]).

The following is the result due to Gaglione and Spellman and independently to Remeslennikov that ties together fully residual freeness and universal freeness.

Theorem 3.3. ([15] [16]) Suppose $G$ is residually free. Then the following 
are equivalent.

(1) $G$ is fully residually free,

(2) $G$ is commutative transitive,

(3) $G$ is universally free if nonabelian.

Parts (1) and (2) of the theorem were due originally to Benjamin Baumslag [17].

Prior to the solution of the Tarski problems, it was asked whether there exist finitely generated non-free elementary free groups. By this it was meant that if all countable nonabelian free groups do have the same first-order theory do there exist finitely generated non-free groups with exactly the same first-order theory as the class of nonabelian free groups.

In the finitely generated case, the answer is yes, and both the KharlampovichMyasnikov solution and the Sela solution provide a complete characterization of the finitely generated elementary free groups. In the Kharlampovich-Myasnikov formulation these are given as a special class of what are termed NTQ groups (see [1] or [2]). The primary examples of non-free elementary free groups are the orientable surface groups of genus $g \geq 2$ and the nonorientable surface groups of genus $g \geq 4$ (see [1]). Recall that a surface group is the fundamental group of a compact surface. If the surface is orientable it is an orientable surface group otherwise a nonorientable surface group.

\section{Universal Equivalence of Group Rings}

We now attempt to extend the Tarski results to group rings, in particular to group rings of free groups. We start by considering the universal theory of a group ring $R[G]$ where $R$ is a commutative ring with an identity. Let $F$ be a nonabelian finitely generated free group and $\mathbb{Z}$ the integers. If $F_{1}$ is any other nonabelian countable free group then we have the same snake eating its tail situation $F_{1} \leq F \leq F_{1}$ as before. Since every subring of $\mathbb{Z}$ with an identity is $\mathbb{Z}$ itself the same argument as for groups shows that for any language we use

$$
T h_{\forall} \mathbb{Z}[F] \subset T h_{\forall} \mathbb{Z}\left[F_{1}\right] \subset T h_{\forall} \mathbb{Z}[F]
$$

It follows as for groups that all integral group rings of finitely generated nonabelian free groups are universally equivalent. Here we will use the universal theory with the axioms $T_{2}$ and language $L_{2}$ for group rings.

Theorem 4.1. All integral group rings for nonabelian countable free groups are universally equivalent.

In this context we call any group ring universally equivalent to $\mathbb{Z}[F]$ for a nonabelian countable free group a universally free group ring. We now consider the question of classifying the universally free group rings in a manner similar to the Gaglione-Spellman-Remeslennikov theorem.

In [18] the following more general results were proved answering this. The proofs are short so they are included. The proofs depend on material in the book by Bell and Slomson [19].

Theorem 4.2. Let $R$ be a commutative ring with identity $1 \neq 0$ and let $G$ 
and $H$ be groups. If $G$ and $H$ are universally equivalent with respect to $L_{0}$, then $R[G]$ and $R[H]$ are universally equivalent with respect to $L_{1}$.

Proof. $H$ is a model of $T h_{\forall}(G)$. Thus, by Lemma 3.8 of Chapter 9 of [19], there is a nonempty set $I$ and an ultrafilter $D$ on $I$ such that $H$ embeds in ${ }^{*} G=G^{I} / D$. Hence, $R[H]$ embeds in ${ }^{*}(R[G]) \equiv R[G]$. Hence, $R[H]$ is a model of $T h_{\forall}(R[G])$ with respect to $L_{1}$. Interchanging, the roles of $G$ and $H$ in the above, we get $R[G]$ is a model of $T h_{\forall}(R[H])$ with respect to $L_{1}$. Therefore, $T h_{\forall}(R[G])=T h_{\forall}(R[H])$ with respect to $L_{1}$.

Theorem 4.3. Let $R$ and $S$ be commutative rings with identity $1 \neq 0$ ad let $G$ be a group. If $R$ and $S$ are universally equivalent with respect to $L_{1}$, then $R[G]$ and $S[G]$ are universally equivalent with respect to $L_{1}$.

The proof of the second theorem is similar to that of the first (see [18]).

Combining these two theorems and using the transitivity of universal equivalence with respect to $L_{1}$, we immediately deduce.

Theorem 4.4. Let $G$ and $H$ be groups and let $R$ and $S$ be commutative rings with identity $1 \neq 0$. If $G$ and $H$ are universally equivalent with respect to $L_{0}$ and $R$ and $S$ are universally equivalent with respect to $L_{1}$, then $R[G]$ and $S[H]$ are universally equivalent with respect to $L_{1}$.

These two theorems can be utilized to handle the classification of universally free group rings which is stated in the following corollary.

Corollary 1. Let $F=\left\langle a_{1}, a_{2} ;\right\rangle$ be the rank 2 free group and let $\mathbb{Z}$ be the ring of integers. Let $G$ be a group and $R$ be a commutative ring with identity $1 \neq 0$. If $G$ is universally equivalent to $F$ with respect to $L_{0}$ and $R$ is universally equivalent to $\mathbb{Z}$ with respect to $L_{1}$, then $R[G]$ is universally equivalent to $\mathbb{Z}[F]$ with respect to $L_{1}$.

In [18] a second independent proof of the above result was given. We want next to extend to group rings an analog of the Gaglione-Spellman-Remeslennikov result tying universal freeness to fully residual freeness. First we need the following idea which is the appropriate analog of residual freeness for rings.

A ring $R$ with identity $1 \neq 0$ which is discriminated by $\mathbb{Z}$ is said to be $\omega$-residually $\mathbb{Z}$. Such rings have characteristic zero so $\mathbb{Z} \leq R$. A ring all of whose finitely generated subrings are $\omega$-residually $\mathbb{Z}$ is said to be locally $\omega$ residually $\mathbb{Z}$. Of course, every ring is the direct union of its finitely generated subrings. Hence, every locally $\omega$-residually $\mathbb{Z}$ ring is universally equivalent to $\mathbb{Z}$. The converse is also known to be true.

Using these concepts we may paraphrase Corollary 1 thusly. Let $F=\left\langle a_{1}, a_{2} ;\right\rangle$ and let $R$ be a locally $\omega$-residually $\mathbb{Z}$ ring. If $G$ is a nonabelian locally fully residually free group, then $R[G]$ is universally equivalent to $\mathbb{Z}[F]$ with respect to $L_{1}$. Now $R[G]$ is the direct union of the family of subrings of the form $R_{0}\left[G_{0}\right]$ where $R_{0}$ is a finitely generated subring of $R$ and $G_{0}$ is a nonabelian finitely generated subgroup of $G$. If $x, y \in G_{0}$ and do not commute, then $\langle x, y\rangle \cong F$. By Lemm 3.5 it suffices to show that such subrings are discriminated by $\mathbb{Z}[F]$.

If $R$ is a ring with identity $1 \neq 0$ we let $U(R)$ be its group of units. If $R$ 
is a commutative ring with identity $1 \neq 0$ and $G$ a group then an element of $U(R[G])$ is said to be a trivial unit provided it has the form $u g$ where $u \in U(R)$ and $g \in G$.

Definition 4.5. A group $G$ is orderable provided it admits a linear order < satisfying the conditions that $h g_{1}<h g_{2}$ whenever $g_{1}<g_{2}$ and $g_{1} h<g_{2} h$ whenever $g_{1}<g_{2}$ as $g_{1}, g_{2}$ and $h$ varyover $G$.

It is well known that free groups are orderable. See e.g. [20]. One can find, for example in Passman's book [21], that, if $K$ is a field and $G$ is an orderable group, then $K[G]$ has trivial units only. From that it easily follows that if $D$ is an integral domain and $G$ is an orderable group, then $D[G]$ has trivial units only. Now suppose that $R$ is any locally $\omega$-residually $\mathbb{Z}$ ring. Then $R$ is universally equivalent to $\mathbb{Z}$ and thus the following four sentences hold in $R$.

(i) $\forall x, y(x y=y x)$.

(ii) $\forall x, y((x y=0) \rightarrow((x=0) \vee(y=0)))$.

(iii) $\forall x y((x y=1) \rightarrow((x=1) \vee(x=-1)))$.

(iv) $1 \neq-1$.

It follows that any locally $\omega$-residually $\mathbb{Z}$ ring $R$ is an integral domain with group of units $U(R)=\{1,-1\}=U(\mathbb{Z})$. Note that since $F=\left\langle a_{1}, a_{2} ;\right\rangle$ is orderable and $\mathbb{Z}$ is an integral domain, $\mathbb{Z}[\mathbb{F}]$ has trivial units only and thus

$$
U(\mathbb{Z}[F])=\langle-1\rangle \times F .
$$

Theorem 4.6. Let $F=\left\langle a_{1}, a_{2} ;\right\rangle, R$ be a locally $\omega$-residually $\mathbb{Z}$ ring and $G$ be a group. If $R[G]$ is universally equivalent to $\mathbb{Z}[F]$ with respect to $L_{1}$, then $G$ is a universally free group.

Lemma 4.7. $U(R[G])$ is universally equivalent to $U(\mathbb{Z}[F])$ with respect to $L_{0}$.

Proof. Suppose that $\forall x_{1}, \cdots, x_{n} \varphi\left(x_{1}, \cdots, x_{n}\right)$ is a universal sentence of $L_{0}$ true in $U(\mathbb{Z}[F])$. Then

$$
\forall x_{1}, \cdots, x_{n}, y_{1}, \cdots, y_{n}\left(\bigwedge_{i=1}^{n}\left(\left(x_{i} y_{i}=1\right) \wedge\left(y_{i} x_{i}=1\right)\right) \rightarrow \varphi\left(x_{1}, \cdots, x_{n}\right)\right)
$$

is true in $\mathbb{Z}[F]$; hence, it is true in $R[G]$ and so $\forall x_{1}, \cdots, x_{n} \varphi\left(x_{1}, \cdots, x_{n}\right)$ holds in $U(R[G])$.

Suppose $\exists x_{1}, \cdots, x_{n} \psi\left(x_{1}, \cdots, x_{n}\right)$ is an existential sentence of $L_{0}$ true in $U(\mathbb{Z}[F])$. Then

$$
\exists x_{1}, \cdots, x_{n}, y_{1}, \cdots, y_{n}\left(\bigwedge_{i=1}^{n}\left(\left(x_{i} y_{i}=1\right) \wedge\left(y_{i} x_{i}=1\right)\right) \wedge \psi\left(x_{1}, \cdots, x_{n}\right)\right)
$$

is true in $U(\mathbb{Z}[F])$; hence, it is true in $R[G]$ and so $\exists x_{1}, \cdots, x_{n} \psi\left(x_{1}, \cdots, x_{n}\right)$ holds in $U(R[G])$. It follows that $U(\mathbb{Z}[F])$ and $U(R[G])$ are universally equivalent with respect to $L_{0}$.

Lemma 4.8. -1 is the unique element of $U(R[G])$ having order 2. Furthermore, -1 is central in $U(R[G])$. 
Proof. The following sentences of $L_{0}$ are true in $\langle-1\rangle \times F=U(\mathbb{Z}[F])$.

$$
\begin{aligned}
& \exists z\left(\left(z^{2}=1\right) \wedge(z \neq 1)\right) \\
& \forall z_{1} z_{2}\left(\left(\left(z_{1}^{2}=1\right) \wedge\left(z_{2}^{2}=1\right) \wedge\left(z_{1} \neq 1\right) \wedge\left(z_{2} \neq 1\right)\right) \rightarrow\left(z_{1}=z_{2}\right)\right) \\
& \forall x, z\left(\left(z^{2}=1\right) \rightarrow(x z=z x)\right) .
\end{aligned}
$$

Hence, they are true in $U(R[G])$ and the conclusions follow.

Lemma 4.9. If $R[G]$ is universally equivalent to $\mathbb{Z}[\mathbb{F}]$ with respect to $L_{1}$, then $G$ is an orderable group. In particular, $R[G]$ has trivial units only and so

$$
U(R[G])=\langle-1\rangle \times G .
$$

Proof. $\langle-1\rangle \times G \leq U(R[G])$ from which we get an embedding

$$
G \rightarrow U(R[G]) /\langle-1\rangle
$$

by factoring out by the central subgroup $\langle-1\rangle$. It is shown in [22] that orderability is captured by a set $S$ of universal sentences of $L_{0}$. Hence,

$$
F \cong U(\mathbb{Z}[F]) /\langle-1\rangle
$$

is a model of $S$.

Let $\sigma \in S$. Suppose $\sigma$ is $\forall x_{1}, \cdots, x_{n} \varphi\left(x_{1}, \cdots, x_{n}\right)$. Let $\sigma^{*}$ be the sentence

$$
\forall x_{1}, \cdots, x_{n}, z\left(\left(z^{2}=1\right) \wedge(z \neq 1)\right) \rightarrow \hat{\varphi}\left(x_{1}, \cdots, x_{n}, z\right)
$$

where $\hat{\varphi}\left(x_{1}, \cdots, x_{n}, z\right)$ is obtained from $\varphi\left(x_{1}, \cdots, x_{n}\right)$ by replacing each equation $u(\bar{x})=1$ with the disjunction $(u(\bar{x})=1) \vee(u(\bar{x})=z)$ and each inequation $w(\bar{x}) \neq 1$ with the conjunction $(w(\bar{x}) \neq 1) \wedge(w(\bar{x}) \neq z)$. Then $\sigma^{*}$ holds in $U(\mathbb{Z}[F])$ since it asserts that $\sigma$ holds in $U(\mathbb{Z}[\mathbb{F}])$ modulo $\langle-1\rangle$. Therefore, $\sigma^{*}$ holds in $U(R[G])$ and so $\sigma$ holds in $U(R[G])$ modulo $\langle-1\rangle$. Therefore, $U(R[G]) /\langle-1\rangle$ is orderable. Since orderability is preserved in subgroups and $G$ embeds in $U(R[G]) /\langle-1\rangle, G$ is orderable.

This far, we have viewed group rings as instances of models of $T_{1}$ in $L_{1}$. If we view group rings as standard models of $T_{2}$ in $L_{2}$ and assert the stronger condition of universal equivalence with respect to $L_{2}$, then the converse to Theorem 4.3 is true (see [18]).

Theorem 4.10. Let $G$ and $H$ be groups and let $R$ and $S$ be commutative rings with identity $1 \neq 0$. View the group rings $R[G]$ and $S[H]$ as standard models of $T_{2}, A$ and $B$ respectively. If $A$ and $B$ are universally equivalent with respect to $L_{2}$, then $G$ is universally equivalent to $H$ with respect to $L_{0}$ and $R$ is universally equivalent to $S$ with respect to $L_{1}$.

Proof. Suppose $\forall x_{1}, \cdots, x_{n} \varphi\left(x_{1}, \cdots, x_{n}\right)$ is a universal sentence of $L_{0}$ true in $G=\Gamma(A)$. Then

$$
\forall x_{1}, \cdots, x_{n}\left({ }_{i=1}^{n} \Gamma\left(x_{i}\right) \rightarrow \varphi\left(x_{1}, \cdots, x_{n}\right)\right)
$$

is true in $R[G]=A$. Therefore it is true in $S[H]=B$ and so $\forall x_{1}, \cdots, x_{n} \varphi\left(x_{1}, \cdots, x_{n}\right)$ is true in $H=\Gamma(B)$. 
Suppose $\exists x_{1}, \cdots, x_{n} \psi\left(x_{1}, \cdots, x_{n}\right)$ is an existential sentence of $L_{0}$ true in $G=\Gamma(A)$. Then

$$
\exists x_{1}, \cdots, x_{n}\left(\bigwedge_{i=1}^{n} \Gamma\left(x_{i}\right) \wedge \psi\left(x_{1}, \cdots, x_{n}\right)\right)
$$

is true in $R[G]=A$. Hence it is true in $S[H]=B$ and so $\exists x_{1}, \cdots, x_{n} \psi\left(x_{1}, \cdots, x_{n}\right)$ is true in $H=\Gamma(B)$. It follows that $G$ and $H$ are universally equivalent with respect to $L_{0}$.

Suppose $\forall x_{1}, \cdots, x_{n} \alpha\left(x_{1}, \cdots, x_{n}\right)$ is a universal sentence of $L_{1}$ true in $R=P(A)$. Then

$$
\forall x_{1}, \cdots, x_{n}\left(\bigwedge_{i=1}^{n} P\left(x_{i}\right) \rightarrow \alpha\left(x_{1}, \cdots, x_{n}\right)\right)
$$

is true in $R[G]=A$. Hence it is true in $S[H]=B$ and so $\forall x_{1}, \cdots, x_{n} \alpha\left(x_{1}, \cdots, x_{n}\right)$ is true in $S=P(B)$.

Suppose $\exists x_{1}, \cdots, x_{n} \beta\left(x_{1}, \cdots, x_{n}\right)$ is an existential sentence of $L_{1}$ true in $R=P(A)$. Then

$$
\exists x_{1}, \cdots, x_{n}\left(\bigwedge_{i=1}^{n} \Gamma\left(x_{i}\right) \wedge \beta\left(x_{1}, \cdots, x_{n}\right)\right)
$$

is true in $R[G]=A$. Hence, it is true in $S[H]=B$ and so $\exists x_{1}, \cdots, x_{n} \beta\left(x_{1}, \cdots, x_{n}\right)$ is true in $S=P(B)$. It follows that $R$ and $S$ are universally equivalent with respect to $L_{1}$.

\section{Elementary Equivalence of Group Rings}

We now examine the elementary equivalence of group rings. We need the following proposition that can be found in the book of Chang and Keisler ([23]).

Proposition 1. (Keisler-Shelah [24]) Let $L$ be a first-order language with equality and $A$ and $B$ be $L$-structures. If $A \equiv_{L} B$, then there is a nonempty set $I$ and an ultrafilter $D$ in $I$ such that the ultrapowers ${ }^{*} A=A^{I} / D$ and ${ }^{*} B=B^{I} / D$ are isomorphic.

This proposition was first proven by Keisler using the Generalized Continuum Hypothesis and subsequently reproven by Shelah without need of that assumption.

Using this we get the following which says that if two group rings are elementary equivalent with respect to $L_{2}$ then the groups are elementary equivalent with respect to $L_{0}$ and the rings are elementary equivalent with respect to $L_{1}$.

Theorem 5.1. Let $G$ and $H$ be groups and $R$ and $S$ be commutative rings with $1 \neq 0$. View the group rings $R[G]$ and $S[H]$ as standard models of $T_{2}, A$ and $B$ respectively. If $A \equiv_{L_{2}} B$, then $G \equiv_{L_{0}} H$ and $R \equiv_{L_{1}} S$.

Proof. By Keisler-Shelah there is a nonempty set $I$ and an ultrafilter $D$ on $I$ such that the ultrapowers ${ }^{*} A=A^{I} / D$ and ${ }^{*} B=B^{I} / D$ are isomorphic. Let $\varphi:{ }^{*} A \rightarrow{ }^{*} B$ be an isomorphism. Then $\varphi$ induces a group isomorphism $\varphi_{\Gamma}$ from $\Gamma\left({ }^{*} A\right)={ }^{*} G=G^{I} / D$ onto $\Gamma\left({ }^{*} B\right)={ }^{*} H=H^{I} / D$ as well as a ring isomorphism $\varphi_{P}$ from $P\left({ }^{*} A\right)={ }^{*} R=R^{I} / D$ onto $P\left({ }^{*} B\right)={ }^{*} S=S^{I} / D:$ Now, 
$G \equiv_{L_{0}}{ }^{*} G \cong{ }^{*} H \equiv_{L_{0}}{ }^{*} H \equiv_{L_{0}} H$. Hence, $G \equiv_{L_{0}} H$. Similarly, $R \equiv_{L_{1}}{ }^{*} R \cong{ }^{*} S \equiv_{L_{1}} S$. Hence $R \equiv_{L_{1}} S$.

In [18] the converse was considered, that is whether or not $G \equiv_{L_{0}} H$ and $R \equiv_{L_{1}} S$ imply that $R[G] \equiv_{L_{2}} S[H]$. The total answer was not settled in that paper but a sufficient condition on group rings was introduced for this to occur (see [18]). This is a finiteness condition since the question is answered affirmatively for finite groups $G$ and $H$.

\section{Summary and Conclusion}

In this note we extended the Tarksi theorems for groups to the collection of group rings. In particular we examined the relationships between the universal and elementary theories of the corresponding groups and rings and the corresponding universal and elementary theory of the formed group ring. In order to do this we considered three first-order languages with equality whose model classes are respectively groups, rings and group rings. Our main results are that if $R[G]$ is elementarily equivalent to $S[H]$ then simultaneously the group $G$ is elementarily equivalent to the group $H$ and the ring $R$ is elementarily equivalent to the ring $S$ with respect to the appropriate languages. This yields the ana$\log$ of the basic Tarksi theorem for groups, that is, that all integral groups rings $Z[F]$ where $F$ is a nonabelian free groups are elementarily equivalent. Further if $G$ is universally equivalent to a nonabelian free group $F$ and $R$ is universally equivalent to the integers $\mathbb{Z}$ then $R[G]$ is universally equivalent to $\mathbb{Z}[F]$ again with respect to an appropriate language.

\section{References}

[1] Fine, B., Gaglione, A.M., Myasnikov, A., Rosenberger, G. and Spellman, D. (2015) The Elementary Theory of Groups. DeGruyter.

[2] Fine, B., Gaglione, A.M., Spellman, D. and Rosenberger, G. (2015) The Tarski Problems and Their Solutions. Advances in Pure Mathematics, 5, 212-231. https://doi.org/10.4236/apm.2015.54023

[3] Kharlamapovich, O. and Myasnikov, A. (2016) Equations over Group Rings. To Appear.

[4] Kharlamapovich, O. and Myasnikov, A. (1998) Irreducible Affine Varieties over a Free Group: I. Irreducibility of Quadratic Equations and Nullstellensatz. Journal of Algebra, 200, 472-516. https://doi.org/10.1006/jabr.1997.7183

[5] Kharlamapovich, O. and Myasnikov, A. (1998) Affine Varieties over a Free Group: II. Systems in Triangular Quasi-Quadratic Form and a Description of Residually Free Groups. Journal of Algebra, 200, 517-569.

[6] Kharlamapovich, O. and Myasnikov, A. (2005) The Implicit Function Theorem over Free groups. Journal of Algebra, 290, 1-203. https://doi.org/10.1016/j.jalgebra.2005.04.001

[7] Kharlamapovich, O. and Myasnikov, A. (2005) Effective JSJ Decompositions. Contemporary Mathematics, 378, 87-211. https://doi.org/10.1090/conm/378/07012

[8] Kharlamapovich, O. and Myasnikov, A. (2006) Elementary Theory of Free NonAbelian Groups. Journal of Algebra, 302, 451-552. https://doi.org/10.1016/j.jalgebra.2006.03.033 
[9] Sela, Z. (2001) Diophantine Geometry over Groups I: Makanin-Razborov Diagrams. Publications Mathématiques de IInstitut des Hautes Études Scientifiques, 93, 31 106. https://doi.org/10.1007/s10240-001-8188-y

[10] Sela, Z. (2003) Diophantine Geometry over Groups II: Completions, Closures and Fromal Solutions. Israel Journal of Mathematics, 104, 173-254. https://doi.org/10.1007/BF02787407

[11] Sela, Z. (2005) Diophantine Geometry over Groups III: Rigid and Solid Solutions. Israel Journal of Mathematics, 147, 1-73. https://doi.org/10.1007/BF02785359

[12] Sela, Z. (2004) Diophantine Geometry over Groups IV: An Iterative Procedure for Validation of a Sentence. Israel Journal of Mathematics, 143, 1-71. https://doi.org/10.1007/BF02803494

[13] Sela, Z. (2005) Diophantine Geometry over Groups V: Quantifier Elimination. Israel Journal of Mathematics, 150, 1-97. https://doi.org/10.1007/BF02785359

[14] Magnus, W., Karrass, A. and Solitar, D. (1966) The Combinatorial Group Theory. Wiley Interscience, Hoboken.

[15] Gaglione, A. and Spellman, D. (1993) Even More Model Theory of Free Groups. In: Corson, J., Dixon, M., Evans, M. and Rohl, F., Eds., Infinite Groups and Group Rings, World Scientific Press, Singapore, 37-40. https://doi.org/10.1142/9789814503723_0004

[16] Remeslennikov, V.N. (1989) 9-Free Groups. Siberian Mathematical Journal, 30, 998-1001.

[17] Baumslag, B. (1967) Residually Free Groups. Proceedings of the London Mathematical Society, 17, 402-408. https://doi.org/10.1112/plms/s3-17.3.402

[18] Fine, B., Gaglione, A.M., Spellman, D. and Rosenberger, G. The Elementary and Universal Theory of Group Rings. Journal of Group Theory.

[19] Bell, J.L. and Slomson, A.B. (1971) Models and Ultra Products: An Introduction. 2nd Revised Printing, North-Holland, Amsterdam.

[20] Neumann, B.H. (1949) On Ordered Groups. American Journal of Mathematics, 71, 1-18. https://doi.org/10.2307/2372087

[21] Passman, D.S. (1985) The Algebraic Structure of Group Rings. Dover, Mineola.

[22] Fine, B., Gaglione, A.M. and Spellman, D. The Class of Orderable Groups Is a Quasi Variety with Undecidable Theory. Preprint.

[23] Chang, C.C. and Keisler, H.J. (1977) Model Theory. 2nd Edition, North-Holland, Amsterdam.

[24] Shelah, S. (1972) Every Two Elementarily Equivalent Models Have Isomorphic U1tra Powers. Israel Journal of Mathematics, 10, 224-233.

https://doi.org/10.1007/BF02771574 
Submit or recommend next manuscript to SCIRP and we will provide best service for you:

Accepting pre-submission inquiries through Email, Facebook, LinkedIn, Twitter, etc. A wide selection of journals (inclusive of 9 subjects, more than 200 journals)

Providing 24-hour high-quality service

User-friendly online submission system

Fair and swift peer-review system

Efficient typesetting and proofreading procedure

Display of the result of downloads and visits, as well as the number of cited articles Maximum dissemination of your research work

Submit your manuscript at: http://papersubmission.scirp.org/

Or contact apm@scirp.org 